Epidemiology and Infection

cambridge.org/hyg

\section{Original Paper}

Cite this article: Wi YM, Rhee JY, Kang $\mathrm{Cl}$, Chung DR, Song JH, Peck KR (2018). Clinical predictors of methicillin-resistance and their impact on mortality associated with Staphylococcus aureus bacteraemia.

Epidemiology and Infection 146, 1326-1336. https://doi.org/10.1017/S0950268818001255

Received: 9 November 2017

Revised: 23 April 2018

Accepted: 24 April 2018

First published online: 21 May 2018

Key words:

Bacteraemia; MRSA; outcome; Staphylococcus aureus

Author for correspondence:

K. R. Peck, E-mail: krpeck@skku.edu

\title{
Clinical predictors of methicillin-resistance and their impact on mortality associated with Staphylococcus aureus bacteraemia
}

\author{
Y. M. Wi ${ }^{1}$, J. Y. Rhee ${ }^{2}$, C. I. Kang ${ }^{3}$, D. R. Chung ${ }^{3}$, J. H. Song ${ }^{3}$ and K. R. Peck ${ }^{3}$
}

${ }^{1}$ Division of Infectious Diseases, Samsung Changwon Hospital, Sungkyunkwan University School of Medicine, Changwon-si, Republic of Korea; ${ }^{2}$ Division of Infectious Diseases, Department of Medicine, Dankook University, Chunan-si, Republic of Korea and ${ }^{3}$ Division of Infectious Diseases, Samsung Medical Center, Sungkyunkwan University School of Medicine, Seoul, Republic of Korea

\begin{abstract}
We investigated the clinical predictors of methicillin-resistance and their impact on mortality in 371 patients with Staphylococcus aureus bacteraemia identified from two prospective multicentre studies. Methicillin resistant S. aureus (MRSA) accounted for $42.2 \%$ of communityonset and $74.5 \%$ of hospital-onset cases. No significant clinical difference was found between patients infected with MRSA vs. methicillin-sensitive S. aureus (MSSA), except that the former were more likely to have had hospital-onset bacteraemia and received antibiotics in the preceding 90 days. After stratifying according to the acquisition site, prior antibiotic use was the only independent predictor of having MRSA in both community-onset and hospital-onset cases. The frequency of inappropriate empirical antibiotic therapy was higher in patients with MRSA than in those with MSSA bacteraemia. However, methicillin resistance was not a predictor of mortality in patients and the clinical characteristics and outcomes of both MRSA and MSSA bacteraemia were similar. This study indicates that there are no definitive clinical or epidemiological risk factors which could distinguish MRSA from MSSA cases with the exception of the previous use of antibiotics for having MRSA bacteraemia, which emphasises the prudent use of glycopeptide treatment of patients at risk for invasive MRSA infections.
\end{abstract}

\section{Introduction}

Staphylococcus aureus is among the most common causes of bloodstream infection (BSI) and is widely recognised as the most important cause of BSI-associated death $[1,2]$ being associated with a high mortality rate, presentation of persistent bacteraemia and frequent spread to distant foci $[3,4]$. Prior to the mid-1990s, methicillin-resistant S. aureus (MRSA) was almost exclusively a healthcare-associated pathogen (HA-MRSA) and infections in the community were nearly always caused by methicillin-susceptible strains (MSSA). However, the end of the twentieth century saw the emergence of community-associated MRSA (CAMRSA) strains in patients without traditional epidemiological risk factors for MRSA infection and/or colonisation [5]. Subsequently, CA-MRSA has spread rapidly into hospital settings and has recently begun replacing pre-existing HA-MRSA strains in healthcare facilities [5-7]. This spread of several different CA-MRSA clones has complicated the epidemiological picture and posed a new challenge for infection prevention and control [8].

Previous studies have suggested that the delay in initiation of anti-MRSA therapy adversely affects the outcome of patients with MRSA bacteraemia, especially those with high Pitt Bacteraemia Scores $[9,10]$ and that vancomycin is inferior to beta-lactams for the treatment of MSSA bacteraemia [11]. As a result, risk factors associated with MRSA infection have been highlighted to optimise antibiotic use when initiating empirical antibiotics against $S$. aureus $[12,13]$. A previous study showed that a patient history of MRSA infection or colonisation, recurrent skin infection and severe community-acquired pneumonia were independent predictors of community-acquired MRSA pneumonia [13]. However, a systematic review of 27 published studies showed that previous admission to hospital and antibiotic use were the only commonly examined risk factors associated with MRSA infection at admission, albeit with varying definitions [14]. The authors emphasised that clinicians and health system planners face many challenges in identifying individuals predisposed to MRSA infection [14]. Therefore, it is important to examine the predictors of MRSA infection and to determine whether the associated factors have changed in recent years.

We compared the clinical features of MSSA and MRSA bacteremia cases with a view to identify factors predictive of MRSA infection. In addition, we evaluated the impact of methicillin resistance on mortality in these patients. 


\section{Methods}

\section{Patients and study design}

The study data were obtained from databases for nationwide surveillance of bacteraemia. All patients with positive blood cultures for $S$. aureus were enrolled at 9 secondary- or tertiary-care hospitals through prospective surveillance studies during two periods from June to September 2011 and June to September 2012. Duplicate isolates were excluded from the analysis. For outcome measures, patients with polymicrobial bacteraemia were also excluded. We identified 371 consecutive patients with confirmed $S$. aureus bacteraemia and patient information was entered on a standardised case report form. The data collected included demographic information, co-morbid diseases and conditions, location of bacteraemia onset, shock at presentation, source of bacteraemia, antibiotic use and surgery within the preceding 90 days and appropriateness of antimicrobial treatment. Echocardiographs were performed for all patients with persistent S. aureus bacteraemia of more than 7 days duration. The study was approved by the Institutional Review Board of Samsung Medical Center and the local review boards.

All isolates were confirmed as $S$. aureus by standard methods and tested for antimicrobial susceptibility at each participating hospital using an automated system for the modified broth microdilution method (Vitek; bioMérieux, Durham, NC, USA, or Microscan; Microscan Systems, Renton, WA, USA) according to the recommendations of the Clinical and Laboratory Standards Institute (2010) [15].

\section{Definitions}

Clinically significant bacteraemia was defined as at least one positive blood culture together with clinical features consistent with systemic inflammatory response syndrome. Catheter-related BSI was defined as growth of $>15$ colony-forming units from the catheter tip in a semiquantitative culture or growth of $S$. aureus from a blood sample drawn from a catheter hub at least $2 \mathrm{~h}$ before MRSA was obtained from a peripheral venous blood sample. Infective endocarditis was defined according to the modified Duke criteria [16]. Neutropaenia was defined as an absolute neutrophil count of $<500 / \mathrm{mm}^{3}$. Isolates defined as 'community-onset' were cultured in the outpatient setting and within $48 \mathrm{~h}$ of hospital admission while 'hospital-onset' isolates were classified as obtained $48 \mathrm{~h}$ after hospitalisation. Community-onset infections were further categorised as community-acquired (CA) or HA. HA infections were defined as previously described [17]. Antibiotic use in preceding 90 days was collected by chart review and through interview with patients and prescribers; prescribed antibiotics was further classified into four classes; glycopeptides, $\beta$-lactams, fluoroquinolones and others. A history of MRSA was determined through a review of each hospital's clinical microbiology record and included both asymptomatic MRSA colonisations and infections in the previous 90 days. The severity of underlying disease was classified according to the McCabe and Jackson criteria: rapidly fatal when death was expected within days or weeks; ultimately fatal when death was expected within months or years; and non-fatal when death was not expected [18]. In addition, Pitt Bacteraemia Scores were determined for all patients [19]. Appropriate antibiotic use was defined as the administration of antibiotics, to which the isolated organism was susceptible in vitro, within $24 \mathrm{~h}$ after the acquisition of blood culture samples. Persistent bacteraemia was defined as persisting for $\geqslant 3$ days after initiation of appropriate antibiotic treatment. The outcome measure was the 30-day all-cause mortality, beginning from the date of the index culture. Deaths occurring after discharge but within the 30-day interval were captured using the National Health Insurance system of the Republic of Korea, which records all deaths.

\section{Statistical analysis}

Discrete data are presented as frequencies and percentages and continuous variables are summarised as the mean \pm standard deviation after the normality of data was tested using the ShapiroWilk normality test. The chi-square $\left(\chi^{2}\right)$ test or Fisher's exact test was used to compare categorical variables as appropriate and two-sample $t$-test was used to compare continuous variables. To identify predictors of methicillin resistance in patients with $S$. aureus bacteraemia, a multivariate logistic regression analysis was used to control for the effects of confounding variables and to identify predictors of mortality, a multivariate Cox proportional hazards regression model was used to control for the effects of confounding variables. Variables with $P$ value $<0.05$ were included in the multivariate analyses. The Hosmer-Lemeshow test was used for goodness of fit for logistic regression models. All $P$ values were 2 -tailed and $P<0.05$ was considered significant. All analyses were performed using SPSS version 21.0 (SPSS Inc., Chicago, IL, USA).

\section{Results \\ Baseline characteristics of patients with S. aureus bacteraemia}

A total of 371 patients met the case definition of $S$. aureus bacteraemia; MSSA was isolated from 155 patients (41.8\%) and MRSA from 216 patients (58.2\%). MRSA accounted for $42.2 \%$ of community-onset and $74.5 \%$ of hospital-onset cases. The clinical characteristics and outcomes of patients with $S$. aureus bacteraemia are summarised in Table 1 . Significant differences in clinical and epidemiological characteristics between MRSA and MSSA were found for several variables; hospital-onset origin $(P<0.001)$, surgery in the preceding 90 days $(P<0.001)$, antibiotic use in the same period $(P<0.001)$, history of MRSA infection $(P=0.002)$, underlying cerebrovascular disease $(P=0.005)$ or haematologic malignancy $(P=0.035)$, undergoing immunosuppressive therapy $(P=0.030)$, presentation with central venous catheterisation $(P<0.001)$ or percutaneous drainage $(P=0.001)$, presentation with acute renal failure $(P<0.001)$, primary bacteraemia $(P=0.049)$ and catheter-related BSI $(P=0.030)$ were risk factors for having MRSA bacteraemia. The multivariate analysis showed that hospital-onset (odds ratio (OR) 2.82, 95\% confidence interval (CI) $1.62-4.91, P<0.001)$ and any antibiotic use in the preceding 90 days (OR 2.47, 95\% CI 1.41-4.34, $P=0.002$ ) were independent predictors for MRSA bacteraemia. The goodness of fit of the final logistic regression model appeared to be satisfactory (Hosmer-Lemeshow statistic, $\chi^{2}=5.627, P=0.689$ ). The frequency of inappropriate empirical therapy was higher in patients with MRSA than MSSA bacteraemia (56.5\% vs. $2.6 \%, P<0.001)$, but 30 -day mortality rates did not differ between the two groups (20.9\% vs. $21.5 \%, P=0.828$ ).

\section{Clinical characteristics and outcomes of MRSA according to primary acquisition site}

The clinical characteristics and outcomes of MRSA among S. aureus bacteraemia patients according to acquisition site are 
Table 1. Clinical predictors and outcomes of methicillin resistance bacteraemia in 371 patients with Staphylococcus aureus bacteraemia

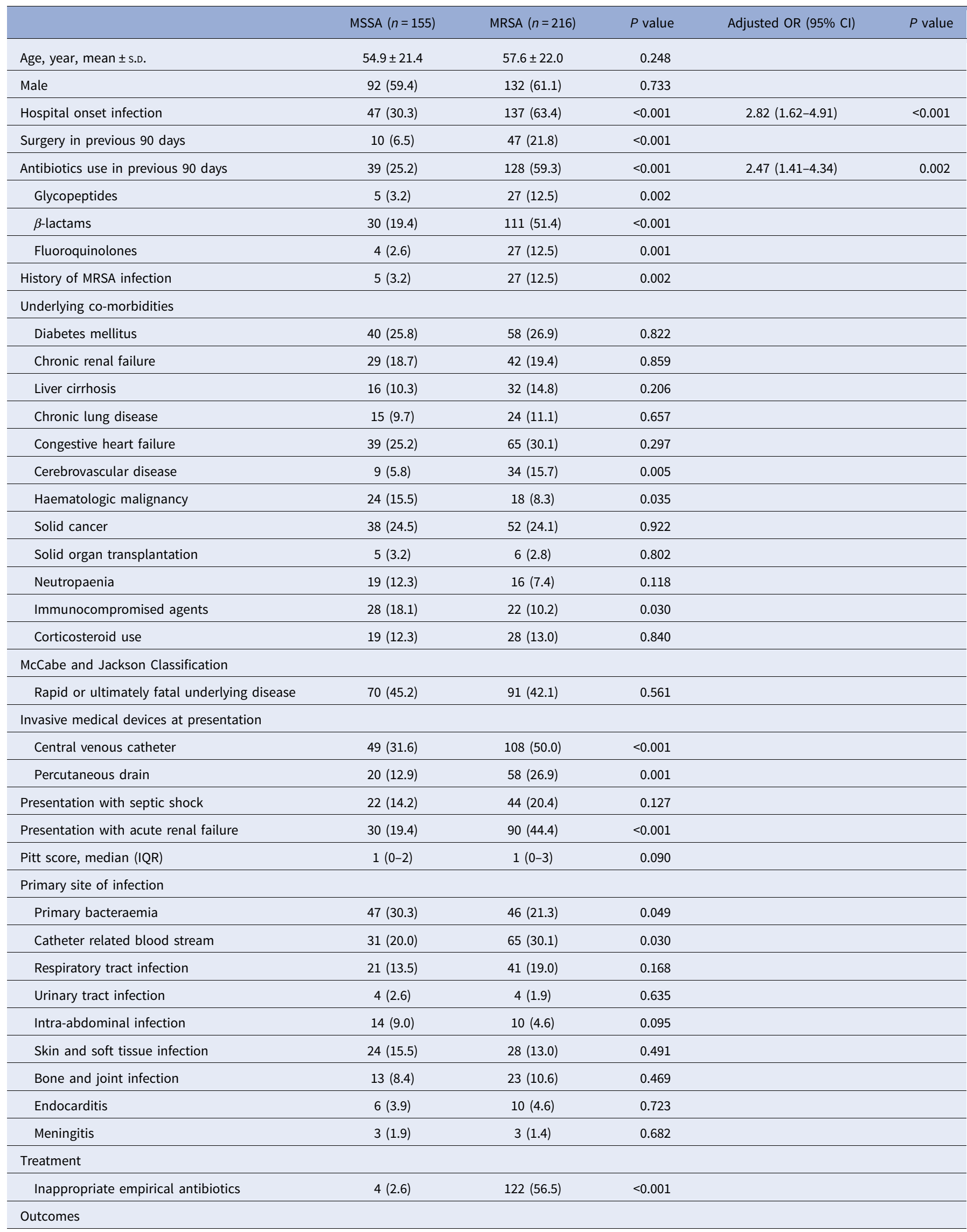


Table 1. (Continued.)

\begin{tabular}{lccc}
\hline & MSSA $(n=155)$ & MRSA $(n=216)$ & $P$ value \\
\hline Persistent bacteraemia & $39(25.2)$ & $80(37.0)$ & 0.326 \\
\hline Mortality & $32(20.9)$ & $47(21.9)$ & 0.828 \\
\hline
\end{tabular}

MSSA, methicillin-susceptible S. aureus; MRSA, methicillin-resistant S. aureus; OR, odds ratio; Cl, confidence interval; s.D., standard deviation; IQR, interquartile range. Hosmer-Lemeshow goodness of fit statistic $\chi^{2}=5.627, P=0.689$

Data are $n(\%)$ unless otherwise stated.

presented in Table 2. Surgery in the previous 90 days $(P=0.007)$, antibiotic use in the same period $(P=0.005)$ and bone and joint infection $(P=0.032)$ were risk factors for CA-MRSA bacteraemia. By multivariate analysis only prior antibiotic use (OR 2.04, 95\% CI 1.01-4.14, $P=0.047$ ) was an independent predictor of MRSA in community-onset cases. Prescribed $\beta$-lactams was the only antibiotic class associated with having MRSA bacteraemia among prior antibiotic uses in both the multivariate analysis and in bivariate testing. The goodness of fit of the final logistic regression model appeared to be satisfactory (HosmerLemeshow statistic, $\left.\chi^{2}=2.701, P=0.259\right)$. On the other hand, for hospital-onset cases, several risk factors were more prevalent in MRSA than MSSA bacteraemia including: surgery in previous 90 days $(P=0.040)$, antibiotic use in the same period $(P<0.001)$, underlying diabetes $(P=0.017)$, underlying cerebrovascular diseases $(P=0.008)$ or haematologic malignancy $(P=0.006)$, neutropaenia $(P=0.035)$, use of immunosuppressive agents $(P=0.001)$, central venous catheterisation $(P=0.030)$ and percutaneous drainage at presentation $(P=0.021)$, presentation with septic shock $(P=0.040)$ or acute renal failure $(P<0.001)$. Prior use of any antibiotic (OR 2.25, 95\% CI 1.01-5.01, $P=0.047$ ) was also the only independent predictor of HA-MRSA bacteremia in multivariate analysis. The goodness of fit of the final logistic regression model in hospital-onset population appeared to be satisfactory (Hosmer-Lemeshow statistic, $\chi^{2}=6.095, P=0.637$ ).

\section{Predictors of 30-day mortality in patients with S. aureus bacteraemia}

The multivariate analysis of potential risk factors associated with 30-day mortality of cases is shown in Table 3. Variables with $P$-value of $<0.05$ in the univariate analysis (i.e. age, liver cirrhosis, solid cancer, rapidly or ultimately fatal disease, central venous catheterisation at presentation, septic shock, acute renal failure, Pitt Bacteraemia Score, respiratory, or skin and soft tissue infection, endocarditis and persistent bacteraemia) were included in the subsequent multivariate analysis. A Cox proportional hazards model revealed that of the above factors, age (hazard ratio $(\mathrm{HR})=$ 1.03 , 95\% CI 1.01-1.04; $P=0.001)$, liver cirrhosis $(\mathrm{HR}=2.21,95 \%$ CI 1.24-3.97, $P=0.008)$, rapidly or ultimately fatal disease $(\mathrm{HR}=$ 2.38, 95\% CI 1.46-3.89, $P=0.001)$, septic shock $(\mathrm{HR}=2.14,95 \%$ CI 1.22-3.78; $P=0.008)$, Pitt Bacteraemia Score $(\mathrm{HR}=1.14,95 \%$ CI $1.05-1.23, P=0.002)$, skin and soft tissue infection $(\mathrm{HR}=0.28$, 95\% CI $0.09-0.90, P=0.032)$ and persistent bacteraemia (HR = 2.84, 95\% CI 1.76-4.56; $P<0.001)$ were independent predictors of 30-day mortality. Methicillin resistance $(\mathrm{HR}=1.01,95 \% \mathrm{CI}$ $0.66-1.53 ; P=0.972$ ) was not a predictor of mortality in these patients and remained non predictive of mortality after stratifying according to acquisition site in both community-onset $(\mathrm{HR}=$ $0.96,95 \%$ CI $0.52-1.77 ; P=0.889)$ and hospital-onset cases $(\mathrm{HR}=1.06,95 \%$ CI $0.54-2.07 ; P=0.862)$ (Table 4$)$.

\section{Discussion}

Our study found no significant differences in clinical outcome between bacteraemic patients infected with MRSA vs. MSSA. However, MRSA patients were more likely to have acquired the infection in hospital and received antibiotics in the preceding 90 days and this was the only marker predictive of methicillinresistance. Inappropriate empirical therapy was more frequently prescribed for MRSA bacteraemia but the 30-day mortality did not differ between the two groups.

The epidemiology of $S$. aureus has undergone significant changes over the past three decades. Initially, HA-MRSA infection was thought to be primarily a HA disease; however, several CAMRSA strains have emerged in the community some of which have recently spread into healthcare settings [5-7]. In addition, a recent study from Chicago, USA showed that a possible role reversal had occurred for MSSA and MRSA strains as the former were predominantly HA and many MRSA patients no longer had recent exposure to the healthcare setting [20]. Therefore, it is important to examine the factors that may be associated with MRSA infection. Miller et al. [21] showed from a prospective investigation of consecutive hospitalised patients with $S$. aureus infection in Los Angeles, that clinical and epidemiological risk factors did not reliably distinguish between MRSA and MSSA. Similar findings were reported from a study of $S$. aureus infections among military veterans except that methicillin resistance was higher in subjects who had a prior MRSA infection or stay in a long-term care facility [22]. Likewise, a cross-sectional study from Colombia found that most clinical and epidemiological factors evaluated did not allow discrimination of MRSA from MSSA infected patients with the exception of a history of MRSA infection, prior antibiotic therapy and stay in children's day care centres [23]. These factors are corroborated by the few clinical differences found in our study between MRSA and MSSA patients, with prior antibiotics being the only independent predictor of both CA- and HA-MRSA bacteraemia. In community-onset $S$. aureus cases, only prior $\beta$-lactam therapy was a risk factor for having MRSA, as opposed to all classes of antibiotics for hospital onset MRSA. In another study, prior exposure to the carbapenems appeared to have a gradient effect for the emergence of methicillin resistance [24].

Other established risk factors $[25,26]$, including history of MRSA infection, residing in a long-term care facility with MRSA infections, recent surgery and indwelling percutaneous medical devices and catheters, failed to differentiate MRSA from MSSA infection in our study. Taken together with other studies, these results support the view that MRSA and MSSA bacteraemic infection share most clinical characteristics. This might be explained by the striking increase of CA-MRSA infections in many countries and their dissemination in to healthcare settings.

Consistent with previous studies [27, 28], the clinical spectrum of disease caused by MRSA appears to be similar to that of MSSA 
Table 2. Clinical predictors and outcomes of patients with Staphylococcus aureus bacteraemia stratified by acquisition site

\begin{tabular}{|c|c|c|c|c|c|c|c|c|c|c|}
\hline & \multicolumn{5}{|c|}{ Community-onset infection } & \multicolumn{5}{|c|}{ Hospital-onset infection } \\
\hline & $\begin{array}{c}\text { MSSA } \\
(n=108)\end{array}$ & $\begin{array}{l}\text { MRSA } \\
(n=79)\end{array}$ & $P$ value & $\begin{array}{l}\text { Adjusted OR } \\
\quad(95 \% \mathrm{Cl})\end{array}$ & $P$ value & $\begin{array}{c}\text { MSSA } \\
(n=47)\end{array}$ & $\begin{array}{c}\text { MRSA } \\
(n=137)\end{array}$ & $P$ value & $\begin{array}{l}\text { Adjusted OR } \\
\quad(95 \% \mathrm{Cl})\end{array}$ & $P$ value \\
\hline Age, year, mean \pm S.D. & $55.9 \pm 21.1$ & $57.7 \pm 21.2$ & 0.573 & & & $52.7 \pm 22.2$ & $57.6 \pm 22.6$ & 0.204 & & \\
\hline Male & $68(63.0)$ & $53(67.1)$ & 0.560 & & & $24(51.1)$ & $79(57.7)$ & 0.432 & & \\
\hline Healthcare risk factor & $54(50.0)$ & $50(63.3)$ & 0.071 & & & NA & NA & NA & & \\
\hline Surgery in previous 90 days & $5(4.6)$ & $13(16.5)$ & 0.007 & & & $5(10.6)$ & $34(24.8)$ & 0.040 & & \\
\hline Antibiotics use in previous 90 days & $20(18.5)$ & $29(36.7)$ & 0.005 & $2.01(1.01-4.14)$ & 0.047 & $19(40.4)$ & $99(72.3)$ & $<0.001$ & $2.25(1.01-5.01)$ & 0.047 \\
\hline Glycopeptides & $2(1.9)$ & 0 & 0.497 & & & $3(6.4)$ & $27(19.7)$ & 0.010 & & \\
\hline$\beta$-lactams & $15(13.9)$ & $27(34.2)$ & 0.004 & & & $15(31.9)$ & $84(61.3)$ & $<0.001$ & & \\
\hline Fluoroquinolones & $3(2.8)$ & $6(7.6)$ & 0.157 & & & $1(2.1)$ & $21(15.3)$ & 0.012 & & \\
\hline History of MRSA infection & $5(4.6)$ & $2(2.5)$ & 0.701 & & & $3(6.4)$ & $22(16.1)$ & 0.095 & & \\
\hline \multicolumn{11}{|l|}{ Underlying co-morbidities } \\
\hline Diabetes mellitus & $35(32.4)$ & $20(25.3)$ & 0.293 & & & $5(10.6)$ & $38(27.7)$ & 0.017 & & \\
\hline Chronic renal failure & $21(19.4)$ & $21(26.6)$ & 0.248 & & & $8(17.0)$ & $21(15.3)$ & 0.783 & & \\
\hline Liver cirrhosis & $14(13.0)$ & $12(15.2)$ & 0.664 & & & $2(4.3)$ & $20(14.6)$ & 0.059 & & \\
\hline Chronic lung disease & $9(8.3)$ & $4(5.1)$ & 0.385 & & & $6(12.8)$ & $20(14.6)$ & 0.756 & & \\
\hline Congestive heart failure & $26(24.1)$ & $23(29.1)$ & 0.439 & & & $13(27.7)$ & $42(30.7)$ & 0.699 & & \\
\hline Cerebrovascular disease & $7(6.5)$ & $5(6.3)$ & 0.967 & & & $2(4.3)$ & $29(21.2)$ & 0.008 & & \\
\hline Haematologic malignancy & $12(11.1)$ & $5(6.3)$ & 0.261 & & & $12(25.5)$ & $13(9.5)$ & 0.006 & & \\
\hline Solid cancer & $22(20.4)$ & $14(17.7)$ & 0.650 & & & $16(34.0)$ & $38(27.7)$ & 0.413 & & \\
\hline Neutropaenia & $9(8.3)$ & $3(3.8)$ & 0.211 & & & $10(21.3)$ & $13(9.5)$ & 0.035 & & \\
\hline Immunocompromised agents & $11(10.2)$ & $4(5.1)$ & 0.203 & & & $17(36.2)$ & $18(13.1)$ & 0.001 & & \\
\hline Corticosteroid use & $7(6.5)$ & $5(6.3)$ & 0.967 & & & $12(25.5)$ & $23(16.8)$ & 0.188 & & \\
\hline \multicolumn{11}{|l|}{ McCabe and Jackson classification } \\
\hline Rapid or ultimately fatal underlying disease & $44(40.7)$ & $34(43.0)$ & 0.753 & & & $26(55.3)$ & $57(41.6)$ & 0.103 & & \\
\hline \multicolumn{11}{|l|}{ Invasive medical devices at presentation } \\
\hline Central venous catheter & $25(23.1)$ & $14(17.7)$ & 0.367 & & & $24(51.1)$ & $94(68.6)$ & 0.030 & & \\
\hline Percutaneous drain & $12(11.1)$ & $10(12.7)$ & 0.746 & & & $8(17.0)$ & $48(35.0)$ & 0.021 & & \\
\hline Presentation with septic shock & $17(15.7)$ & $10(12.7)$ & 0.554 & & & $5(10.6)$ & $34(24.8)$ & 0.040 & & \\
\hline Presentation with acute renal failure & $18(16.7)$ & $11(13.9)$ & 0.609 & & & $12(25.5)$ & $85(62.0)$ & $<0.001$ & & \\
\hline Pitt score, median (IQR) & $1(0-1.8)$ & $1(0-2.0)$ & 0.342 & & & $0(0-2.0)$ & $1(0-3.5)$ & 0.185 & & \\
\hline Primary site of infection & & & & & & & & & & \\
\hline
\end{tabular}




\begin{tabular}{|c|c|c|c|c|c|c|}
\hline Primary bacteraemia & $33(30.6)$ & $20(25.3)$ & 0.432 & $14(29.8)$ & $26(19.0)$ & 0.121 \\
\hline Catheter related blood stream & $15(13.9)$ & $7(8.9)$ & 0.292 & $16(34.0)$ & $58(42.3)$ & 0.317 \\
\hline Respiratory tract infection & $14(13.0)$ & $9(11.4)$ & 0.747 & $7(14.9)$ & $32(23.4)$ & 0.221 \\
\hline Urinary tract infection & $4(3.7)$ & $2(2.5)$ & 1.000 & 0 & $2(1.5)$ & 1.000 \\
\hline Intra-abdominal infection & $12(11.1)$ & $4(5.1)$ & 0.144 & $2(4.3)$ & $6(4.4)$ & 1.000 \\
\hline Skin and soft tissue infection & $17(15.7)$ & $14(17.7)$ & 0.719 & $7(14.9)$ & $14(10.2)$ & 0.384 \\
\hline Bone and joint infection & $12(11.1)$ & $18(22.8)$ & 0.032 & $1(2.1)$ & $5(3.6)$ & 1.000 \\
\hline Endocarditis & $6(5.6)$ & $6(7.6)$ & 0.574 & 0 & $4(2.9)$ & 0.574 \\
\hline Meningitis & $3(2.8)$ & $2(2.5)$ & 1.000 & 0 & $1(0.7)$ & 1.000 \\
\hline \multicolumn{7}{|l|}{ Treatment } \\
\hline Inappropriate empirical antibiotics & $4(3.7)$ & $43(54.4)$ & $<0.001$ & 0 & $79(57.7)$ & $<0.001$ \\
\hline \multicolumn{7}{|l|}{ Outcomes } \\
\hline Persistent bacteremia & $27(25.0)$ & $26(32.9)$ & 0.236 & $12(25.5)$ & $54(39.4)$ & 0.087 \\
\hline Mortality & $22(20.5)$ & $16(20.5)$ & 0.968 & $10(21.3)$ & $31(22.6)$ & 0.848 \\
\hline
\end{tabular}

MSSA, methicillin-susceptible S. aureus; MRSA, methicillin-resistant S. aureus; s.D., standard deviation; IQR, interquartile range.

Hosmer-Lemeshow goodness of fit statistic for community-onset infection $\chi^{2}=2.701, P=0.259$.

Hosmer-Lemeshow goodness of fit statistic for hospital-onset infection $\chi^{2}=6.095, P=0.637$.

Data are $n(\%)$ unless otherwise stated. 
Table 3. Variables associated with 30-day mortality in 371 patients with Staphylococcus aureus bacteraemia

\begin{tabular}{|c|c|c|c|c|}
\hline & \multicolumn{2}{|c|}{ Univariate analysis } & \multicolumn{2}{|c|}{ Multivariate analysis } \\
\hline & $\mathrm{HR}(95 \% \mathrm{Cl})$ & $P$ & Adjusted HR (95\% Cl) & $P$ \\
\hline Male & $1.11(0.71-1.73)$ & 0.662 & & \\
\hline Hospital onset infection & $1.14(0.67-1.95)$ & 0.632 & & \\
\hline Surgery in previous 90 days & $0.84(0.45-1.59)$ & 0.597 & & \\
\hline Antibiotics use in previous 90 days & $1.06(0.68-1.65)$ & 0.785 & & \\
\hline History of MRSA infection & $1.00(0.52-1.94)$ & 0.991 & & \\
\hline \multicolumn{5}{|l|}{ Underlying co-morbidities } \\
\hline Chronic lung disease & $1.29(0.68-2.45)$ & 0.430 & & \\
\hline Congestive heart failure & $1.07(0.66-1.73)$ & 0.796 & & \\
\hline \multicolumn{5}{|l|}{ Cerebrovascular disease } \\
\hline Haematologic malignancy & $0.70(0.32-1.52)$ & 0.363 & & \\
\hline Solid cancer & $1.98(1.25-3.12)$ & 0.004 & & \\
\hline Neutropaenia & $1.30(0.67-2.52)$ & 0.443 & & \\
\hline Immunocompromised agents & $0.56(0.26-1.22)$ & 0.145 & & \\
\hline Corticosteroid use & $0.75(0.38-1.51)$ & 0.424 & & \\
\hline \multicolumn{5}{|l|}{ McCabe and Jackson classification } \\
\hline Presentation with acute renal failure & $2.15(1.38-3.34)$ & 0.001 & & \\
\hline Pitt score & $1.25(1.18-1.33)$ & $<0.001$ & $1.14(1.05-1.23)$ & 0.002 \\
\hline \multicolumn{5}{|l|}{ Primary site of infection } \\
\hline Primary bacteraemia & $1.19(0.70-2.04)$ & 0.527 & & \\
\hline Catheter related blood stream & $0.73(0.43-1.25)$ & 0.248 & & \\
\hline Respiratory tract infection & $2.19(1.36-3.54)$ & 0.001 & & \\
\hline Urinary tract infection & $1.06(0.39-2.90)$ & 0.913 & & \\
\hline Intra-abdominal infection & $0.61(0.19-1.94)$ & 0.402 & & \\
\hline Skin and soft tissue infection & $0.20(0.06-0.64)$ & 0.006 & $0.28(0.09-0.90)$ & 0.032 \\
\hline Bone and joint infection & $0.57(0.25-1.32)$ & 0.192 & & \\
\hline Endocarditis & $2.64(1.32-5.29)$ & 0.006 & & \\
\hline Meningitis & $0.63(0.09-4.55)$ & 0.649 & & \\
\hline Inappropriateness of initial empirical agents & $1.22(0.77-1.91)$ & 0.402 & & \\
\hline Persistent bacteremia & $4.10(2.60-6.44)$ & $<0.001$ & $2.84(1.76-4.56)$ & $<0.001$ \\
\hline
\end{tabular}

HR, hazard ratio; MRSA, methicillin-resistant S. aureus. 
Table 4. Variables associated with 30-day mortality in 371 patients with Staphylococcus aureus bacteraemia stratified by acquisition site

\begin{tabular}{|c|c|c|c|c|c|c|c|c|}
\hline & \multicolumn{4}{|c|}{ Community onset infection } & \multicolumn{4}{|c|}{ Hospital onset infection } \\
\hline & \multicolumn{2}{|c|}{ Univariate analysis } & \multicolumn{2}{|c|}{ Multivariate analysis } & \multicolumn{2}{|c|}{ Univariate analysis } & \multicolumn{2}{|c|}{ Multivariate analysis } \\
\hline & $\mathrm{HR}(95 \% \mathrm{Cl})$ & $P$ & Adjusted HR $(95 \% \mathrm{Cl})$ & $P$ & $\mathrm{HR}(95 \% \mathrm{Cl})$ & $P$ & Adjusted HR $(95 \% \mathrm{Cl})$ & $P$ \\
\hline Age & $1.03(1.01-1.06)$ & 0.001 & $1.04(1.01-1.06)$ & 0.004 & $1.02(1.01-1.03)$ & 0.033 & $1.03(1.01-1.06)$ & 0.003 \\
\hline Male, $n(\%)$ & $1.26(0.68-2.33)$ & 0.466 & & & $0.85(0.49-1.48)$ & 0.563 & & \\
\hline Methicillin resistance & $0.96(0.52-1.77)$ & 0.889 & & & $1.06(0.54-2.07)$ & 0.862 & & \\
\hline Surgery in previous 90 days & $0.18(0.03-1.32)$ & 0.092 & & & $1.04(0.54-2.03)$ & 0.904 & & \\
\hline Antibiotics use in previous 90 days & $1.17(0.61-2.25)$ & 0.640 & & & $1.71(0.90-3.27)$ & 0.103 & & \\
\hline History of MRSA infection & $1.94(0.60-6.27)$ & 0.271 & & & $1.34(0.67-2.68)$ & 0.402 & & \\
\hline \multicolumn{9}{|l|}{ Underlying co-morbidities } \\
\hline Diabetes mellitus & $1.24(0.67-2.31)$ & 0.498 & & & $0.81(0.42-1.57)$ & 0.523 & & \\
\hline Chronic renal failure & $1.72(0.87-3.38)$ & 0.118 & & & $0.77(0.35-1.71)$ & 0.517 & & \\
\hline Liver cirrhosis & $2.05(1.01-4.17)$ & 0.049 & $3.36(1.34-8.38)$ & 0.010 & $1.57(0.74-3.33)$ & 0.243 & & \\
\hline Chronic lung disease & $2.62(1.10-6.22)$ & 0.029 & & & $0.98(0.46-2.08)$ & 0.953 & & \\
\hline Congestive heart failure & $1.29(0.67-2.48)$ & 0.448 & & & $0.87(0.47-1.60)$ & 0.649 & & \\
\hline Cerebrovascular disease & $0.87(0.21-3.61)$ & 0.848 & & & $0.85(0.40-1.80)$ & 0.669 & & \\
\hline Haematologic malignancy & $1.15(0.41-3.22)$ & 0.793 & & & $0.86(0.39-1.91)$ & 0.709 & & \\
\hline Solid cancer & $1.91(0.97-3.73)$ & 0.060 & & & $1.71(0.99-3.05)$ & 0.053 & & \\
\hline Neutropaenia & $1.89(0.67-5.32)$ & 0.226 & & & $1.30(0.63-2.67)$ & 0.475 & & \\
\hline Immunocompromised agents & $0.88(0.27-2.85)$ & 0.832 & & & $0.60(0.27-1.34)$ & 0.212 & & \\
\hline Corticosteroid use & $1.55(0.55-4.36)$ & 0.404 & & & $0.75(0.37-1.54)$ & 0.433 & & \\
\hline \multicolumn{9}{|l|}{ McCabe and Jackson classification } \\
\hline Rapid or ultimately fatal underlying disease & $4.58(2.33-8.99)$ & $<0.001$ & $2.63(1.22-5.67)$ & 0.014 & $2.18(1.25-3.81)$ & 0.006 & $3.80(1.83-7.90)$ & $<0.001$ \\
\hline \multicolumn{9}{|l|}{ Invasive medical devices at presentation } \\
\hline Central venous catheter & $3.12(1.68-5.78)$ & $<0.001$ & & & $1.15(0.65-2.06)$ & 0.634 & & \\
\hline Percutaneous drain & $0.66(0.20-2.13)$ & 0.485 & & & $1.23(0.70-2.16)$ & 0.478 & & \\
\hline Presentation with septic shock & $3.59(1.89-6.83)$ & $<0.001$ & & & $3.08(1.77-5.37)$ & $<0.001$ & & \\
\hline Presentation with acute renal failure & $4.28(2.26-8.09)$ & $<0.001$ & & & $1.71(0.97-3.00)$ & 0.064 & & \\
\hline Pitt score & $1.28(1.19-1.38)$ & $<0.001$ & & & $1.21(1.12-1.31)$ & $<0.001$ & & \\
\hline \multicolumn{9}{|l|}{ Primary site of infection } \\
\hline Primary bacteraemia & $0.95(0.45-1.99)$ & 0.892 & & & $1.13(0.58-2.20)$ & 0.719 & & \\
\hline Catheter related blood stream & $1.12(0.44-2.84)$ & 0.817 & & & $0.63(0.35-1.13)$ & 0.123 & & \\
\hline
\end{tabular}




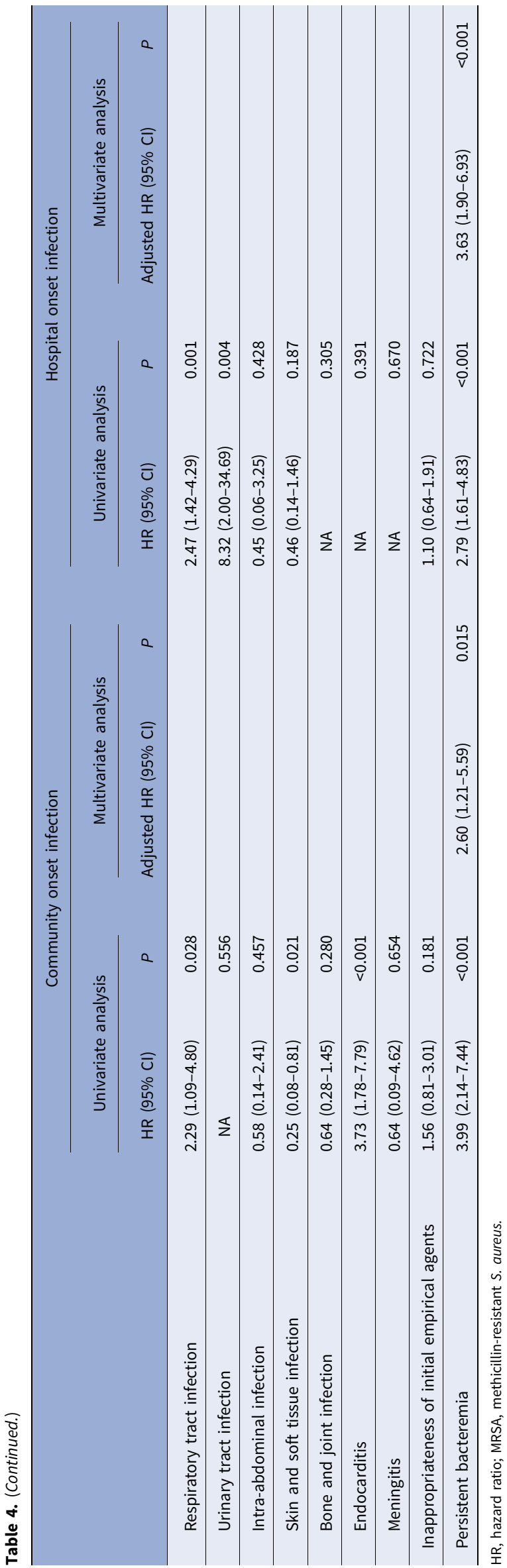

in the community. However, Lee et al. [29] suggested that bone and joint infection was a predictor of CA-MRSA bacteraemia in Korea and identified a specific clone, ST72-SCCmec IV/IVA, which predominated in primary bone and joint infections that progressed to bacteraemia. In our study, bone and joint infections were significantly more prevalent in patients with MRSA than those with community-onset MSSA but statistical significance was lost after adjusting for prior surgery and antibiotic use. The predominance of bone and joint infections in patients with CA-MRSA bacteraemia warrants further investigation.

Many previous studies evaluating the outcomes of MRSA bacteraemia have shown conflicting results [30-33]. Some have reported significant association of MRSA with mortality $[30,32]$, while others have not [31, 33-35]. In the Cox regression model, we could not link MRSA bacteraemia with higher mortality than that with MSSA and after stratifying by acquisition site, methicillin resistance was still not a significant predictor of mortality. Outcomes of MRSA infections could also be made worse due to confounding associations with other prognostic factors. Almost all relevant studies have shown that infections due to MRSA occur in sicker patients $[35,36]$, suggesting that differences in mortality may, at least in part, be attributable to the patients' underlying illnesses. Our study population showed few significant clinical differences between MRSA and MSSA bacteraemia and this was reflected in their similar mortality rates. Indeed, previous studies of patient outcomes with MRSA bacteraemia also linked this to a longer hospital stay and not with mortality $[37,38]$.

We have shown that patients with MRSA bacteraemia were more likely to receive inappropriate empirical antibiotic therapy but this was not an independent predictor of mortality. Others have reported conflicting findings on this issue [39-44]. Interestingly, three studies conducted in Asia found that this factor did not have a detrimental effect on mortality in MRSA bacteraemic patients [43, 45, 46]. Yoon et al. [46] in Korea considered that an initial delay in the use of definitive antibiotics did not necessarily prejudice the clinical outcomes of patients with HA-MRSA bacteraemia and recommended the prudent use of glycopeptides as empirical therapy in the context of increasing antibiotic resistance. Similarly, in Taiwan, Fang et al. [45] did not support the view that earlier empirical use of glycopeptide therapy reduces mortality in patients with HA-MRSA bacteraemia and, likewise, no significant difference in MRSA-related mortality was evident between patients who had received an appropriate or inappropriate empirical regimen [43].

Some limitations of this study are noted. First, only measured variables were controlled and some additional confounding factors were not accounted for such as prior residence in a long-term care facility, or receipt of hemodialysis and hospitalisation, which are often cited in similar studies as contributory factors differentiating between MRSA and MSSA patients. Second, the failure to show a significant difference in mortality according to empirical antibiotic therapy may be a consequence of the limited sample size; third, and we chose to define appropriate antibiotic use based on in vitro susceptibility of the recovered isolate and did not take account of the fact that different agents differ in clinical outcomes such as the reported inferiority of vancomycin to betalactams for the treatment of MSSA bacteraemia [11,44]. Finally, the absence of molecular analysis did not allow investigation of the clonal background which might have impacted on mortality given the reported associations of, for example, strain lineages CC22 and endocarditis, [47], CC8 and vancomycin resistance [48] and CC5 or CC30 and haematogenous complications [49]. 
The predominant CA-MRSA clone in Korea, ST72-SCCmecIV, was shown to be independently associated with lower mortality compared with ST5-SCCmecII [50]; furthermore, the lack of virulence markers including staphylococcal superantigen genes, may play a role in the lower virulence of ST72-SCCmecIV strain [50]. Further clonal analysis of both MRSA and MSSA strains is therefore necessary to explore further any associations of strain type with mortality.

In conclusion, we found no definitive clinical or epidemiological risk factors which could distinguish MRSA from MSSA in bacteraemic patients with the exception of the previous use of antibiotics for having MRSA bacteraemia in such patients regardless of its acquisition site. MRSA bacteraemia was not associated with higher risks of mortality. Finally, the prudent use of glycopeptide treatment of patients at risk for invasive MRSA infections should be emphasised in order to stem the tide of increasing resistance to these highly effective agents.

Conflict of interest. None.

\section{References}

1. Uslan DZ et al. (2007) Age- and sex-associated trends in bloodstream infection: a population-based study in Olmsted County, Minnesota. Archives of Internal Medicine 167, 834-839.

2. Laupland KB et al. (2004) Severe bloodstream infections: a populationbased assessment. Critical Care Medicine 32, 992-997.

3. Khatib R et al. (2009) Persistent Staphylococcus aureus bacteremia: incidence and outcome trends over time. Scandinavian Journal of Infectious Diseases 41, 4-9.

4. van Hal SJ et al. (2012) Predictors of mortality in Staphylococcus aureus bacteremia. Clinical Microbiology Reviews 25, 362-386.

5. David MZ and Daum RS (2010) Community-associated methicillinresistant Staphylococcus aureus: epidemiology and clinical consequences of an emerging epidemic. Clinical Microbiology Reviews 23, 616-687.

6. Song JH et al. (2011) Spread of methicillin-resistant Staphylococcus aureus between the community and the hospitals in Asian countries: an ANSORP study. Journal of Antimicrobial Chemotherapy 66, 1061-1069.

7. Brady JM et al. (2007) Sporadic "transitional" community-associated methicillin-resistant Staphylococcus aureus strains from health care facilities in the United States. Journal of Clinical Microbiology 45, 2654-2661.

8. Cho SY and Chung DR. (2017) Infection prevention strategy in hospitals in the era of community-associated methicillin-resistant Staphylococcus aureus in the Asia-Pacific region: a review. Clinical Infectious Diseases; 64(Suppl. 2), S82-S90.

9. Wi YM et al. (2012) High vancomycin minimum inhibitory concentration is a predictor of mortality in meticillin-resistant Staphylococcus aureus bacteraemia. International Journal of Antimicrobial Agents 40, 108-113.

10. Paul M et al. (2010) Importance of appropriate empirical antibiotic therapy for methicillin-resistant Staphylococcus aureus bacteraemia. Journal of Antimicrobial Chemotherapy 65, 2658-2665.

11. Kim SH et al. (2008) Outcome of vancomycin treatment in patients with methicillin-susceptible Staphylococcus aureus bacteremia. Antimicrobial Agents and Chemotherapy 52, 192-197.

12. Chalmers JD et al. (2014) Healthcare-associated pneumonia does not accurately identify potentially resistant pathogens: a systematic review and meta-analysis. Clinical Infectious Diseases 58, 330-339.

13. Aliberti S et al. (2016) Global initiative for meticillin-resistant Staphylococcus aureus pneumonia (GLIMP): an international, observational cohort study. Lancet Infectious Diseases 16, 1364-1376.

14. Forster AJ et al. (2013) Patient-level factors associated with methicillinresistant Staphylococcus aureus carriage at hospital admission: a systematic review. American Journal of Infection Control 41, 214-220.

15. CLSI (2010) Performance Standards for Antimicrobial Susceptibility Testing; Twentieth Informational Supplement. CLSI document M100S20. Wayne, PA: Clinical and Labortory Stanadards Institute.
16. Li JS et al. (2000) Proposed modifications to the Duke criteria for the diagnosis of infective endocarditis. Clinical Infectious Diseases 30, 633-638.

17. Friedman ND et al. (2002) Health care - associated bloodstream infections in adults: a reason to change the accepted definition of community-acquired infections. Annals of Internal Medicine 137, 791-797.

18. McCabe WR (1974) Gram-negative bacteremia. Advances in Internal Medicine 19, 135-158.

19. Paterson DL et al. (2004) Antibiotic therapy for Klebsiella pneumoniae bacteremia: implications of production of extended-spectrum betalactamases. Clinical Infectious Diseases 39, 31-37.

20. David MZ et al. (2011) Methicillin-susceptible Staphylococcus aureus as a predominantly healthcare-associated pathogen: a possible reversal of roles? PLoS One 6, e18217.

21. Miller LG et al. (2007) Clinical and epidemiologic characteristics cannot distinguish community-associated methicillin-resistant Staphylococcus aureus infection from methicillin-susceptible $S$. aureus infection: a prospective investigation. Clinical Infectious Diseases 44, 471-482.

22. McCarthy NL et al. (2010) Risk factors associated with methicillin resistance among Staphylococcus aureus infections in veterans. Infection Control and Hospital Epidemiology 31, 36-41.

23. Jimenez JN et al. (2013) A comparison of methicillin-resistant and methicillin-susceptible Staphylococcus aureus reveals no clinical and epidemiological but molecular differences. International Journal of Medical Microbiology 303, 76-83.

24. Arias-Ortiz PM et al. (2016) Risk factors for methicillin-resistant Staphylococcus aureus bacteremia: a multicenter matched case-control study. Biomedica: Revista del Instituto Nacional de Salud 36, 612-619.

25. Lowy FD (1998) Staphylococcus aureus infections. New England Journal of Medicine 339, 520-532.

26. Brumfitt W and Hamilton-Miller J (1989) Methicillin-resistant Staphylococcus aureus. New England Journal of Medicine 320, 1188-1196.

27. Kaplan SL et al. (2005) Three-year surveillance of community-acquired Staphylococcus aureus infections in children. Clinical Infectious Diseases 40, 1785-1791.

28. Fridkin SK et al. (2005) Methicillin-resistant Staphylococcus aureus disease in three communities. New England Journal of Medicine 352, 1436-1444.

29. Lee JY et al. (2014) Bone and joint infection as a predictor of communityacquired methicillin-resistant Staphylococcus aureus bacteraemia: a comparative cohort study. Journal of Antimicrobial Chemotherapy 69, 1966-1971.

30. Yilmaz M et al. (2016) Mortality predictors of Staphylococcus aureus bacteremia: a prospective multicenter study. Annals of Clinical Microbiology and Antimicrobials 15, 7.

31. Cosgrove SE et al. (2005) The impact of methicillin resistance in Staphylococcus aureus bacteremia on patient outcomes: mortality, length of stay, and hospital charges. Infection Control and Hospital Epidemiology 26, 166-174

32. Blot SI et al. (2002) Outcome and attributable mortality in critically Ill patients with bacteremia involving methicillin-susceptible and methicillinresistant Staphylococcus aureus. Archives of Internal Medicine 162, 22292235.

33. Mylotte JM and Tayara A (2000) Staphylococcus aureus bacteremia: predictors of 30-day mortality in a large cohort. Clinical Infectious Diseases 31, 1170-1174.

34. Castillo JS et al. (2012) Mortality among critically ill patients with methicillin-resistant Staphylococcus aureus bacteremia: a multicenter cohort study in Colombia. Revista Panamericana de Salud Publica 32, 343-350.

35. Yaw LK, Robinson JO and Ho KM (2014) A comparison of long-term outcomes after meticillin-resistant and meticillin-sensitive Staphylococcus aureus bacteraemia: an observational cohort study. Lancet Infectious Diseases 14, 967-975.

36. Cosgrove SE et al. (2003) Comparison of mortality associated with methicillin-resistant and methicillin-susceptible Staphylococcus aureus bacteremia: a meta-analysis. Clinical Infectious Diseases 36, 53-59.

37. Lodise TP and McKinnon PS (2005) Clinical and economic impact of methicillin resistance in patients with Staphylococcus aureus bacteremia. Diagnostic Microbiology and Infectious Disease 52, 113-122. 
38. Reed SD et al. (2005) Costs and outcomes among hemodialysisdependent patients with methicillin-resistant or methicillin-susceptible Staphylococcus aureus bacteremia. Infection Control and Hospital Epidemiology 26, 175-183.

39. Gasch O et al. (2013) Predictive factors for mortality in patients with methicillin-resistant Staphylococcus aureus bloodstream infection: impact on outcome of host, microorganism and therapy. Clinical Microbiology and infection 19, 1049-1057.

40. Gasch O et al. (2013) Predictive factors for early mortality among patients with methicillin-resistant Staphylococcus aureus bacteraemia. Journal of Antimicrobial Chemotherapy 68, 1423-1430.

41. Marchaim D et al. (2010) Case-control study to identify factors associated with mortality among patients with methicillin-resistant Staphylococcus aureus bacteraemia. Clinical Microbiology and Infection 16, 747-752.

42. Lodise TP et al. (2003) Outcomes analysis of delayed antibiotic treatment for hospital-acquired Staphylococcus aureus bacteremia. Clinical Infectious Diseases 36, 1418-1423.

43. Kim SH et al. (2004) Outcome of inappropriate initial antimicrobial treatment in patients with methicillin-resistant Staphylococcus aureus bacteraemia. Journal of Antimicrobial Chemotherapy 54, 489-497.

44. Khatib R et al. (2006) Impact of initial antibiotic choice and delayed appropriate treatment on the outcome of Staphylococcus aureus bacteremia. European Journal of Clinical Microbiology \& Infectious Diseases 25, 181-185.

45. Fang CT et al. (2006) Early empirical glycopeptide therapy for patients with methicillin-resistant Staphylococcus aureus bacteraemia: impact on the outcome. Journal of Antimicrobial Chemotherapy 57, 511-519.

46. Yoon YK et al. (2016) Effects of inappropriate empirical antibiotic therapy on mortality in patients with healthcare-associated methicillinresistant Staphylococcus aureus bacteremia: a propensity-matched analysis. BMC Infectious Diseases 16, 331.

47. Miller CE et al. (2012) An association between bacterial genotype combined with a high-vancomycin minimum inhibitory concentration and risk of endocarditis in methicillin-resistant Staphylococcus aureus bloodstream infection. Clinical Infectious Diseases 54, 591-600.

48. Holmes NE et al. (2014) Genetic and molecular predictors of high vancomycin MIC in Staphylococcus aureus bacteremia isolates. Journal of Clinical Microbiology 52, 3384-3393.

49. Fowler Jr. VG et al. (2007) Potential associations between hematogenous complications and bacterial genotype in Staphylococcus aureus infection. Journal of Infectious Diseases 196, 738-747.

50. Park KH et al. (2015) Community-associated MRSA strain ST72 SCCmecIV causing bloodstream infections: clinical outcomes and bacterial virulence factors. Journal of Antimicrobial Chemotherapy 70, 1185-1192. 